\title{
Asymptotic Formulas Related to Free Products of Cyclic Groups
}

\author{
By Morris Newman
}

\begin{abstract}
Asymptotic formulas for the number of subgroups of a given index of the free product of finitely many cyclic groups are given. The classical modular group $\Gamma$ is discussed in detail, and a table of the number of subgroups of $\Gamma$ of index $n$ is given for $1 \leqslant n \leqslant 100$.
\end{abstract}

Formulas for the number of subgroups of a given index of free groups of finite rank have been given by $\mathbf{M}$. Hall [2], and have been generalized to the case of free products of finitely many cyclic groups by I. M. S. Dey [1]. In this note we consider the asymptotic behavior of these numbers, and also give some tabular material for the case of the classical modular group.

These formulas have the common feature that the recurrence formulas associated with them have the same structure; and before considering questions of asymptotic behavior, we consider the formulas from a purely formal point of view.

Let $\alpha_{0}, \alpha_{1}, \alpha_{2}, \ldots, M_{1}, M_{2}, M_{3}, \ldots$ be sequences of real numbers such that

$$
\alpha_{0}=1, \quad \sum_{k=1}^{n} \alpha_{n-k} M_{k}=n \alpha_{n}, \quad n \geqslant 1
$$

Define the formal power series $f(x), g(x)$ by

$$
f(x)=\sum_{n=0}^{\infty} \alpha_{n} x^{n}, \quad g(x)=\sum_{n=1}^{\infty} M_{n} x^{n} .
$$

Then (1) is equivalent to the identity

$$
g(x)=x f^{\prime}(x) / f(x)
$$

Formula (2) implies that

$$
\sum_{n=1}^{\infty} \frac{M_{n}}{n} x^{n}=\log f(x)
$$

so that

$$
\sum_{n=1}^{\infty} \frac{M_{n}}{n} x^{n}=\log (1+f(x)-1)=\sum_{n=1}^{\infty} \frac{(-1)^{n-1}}{n}(f(x)-1)^{n} .
$$

Comparing coefficients of corresponding powers of $x$ in (4), we find the following result, which we state as a theorem:

THEOREM 1. The numbers $M_{n}$ are given explicitly as functions of the numbers $\alpha_{n}$ by the formula

Received March 18, 1976.

AMS (MOS) subject classifications (1970). Primary 20E35, 10D05, 10-04.

Key words and phrases. Free products, cyclic groups, free groups, classical modular group, asymptotic formulas, tables. 


$$
\frac{M_{n}}{n}=\sum_{k=1}^{n} \frac{(-1)^{k-1}}{k} S_{k}
$$

where

$$
S_{k}=\sum_{n_{1}+n_{2}+\cdots+n_{k}=n ; n_{i} \geqslant 1} \alpha_{n_{1}} \alpha_{n_{2}} \cdots \alpha_{n_{k}} .
$$

Conversely, if we rewrite (4) as

$$
f(x)=\exp \left(\sum_{n=1}^{\infty} \frac{M_{n}}{n} x^{n}\right)=\prod_{n=1}^{\infty} \exp \left(\frac{M_{n}}{n} x^{n}\right)
$$

we find that

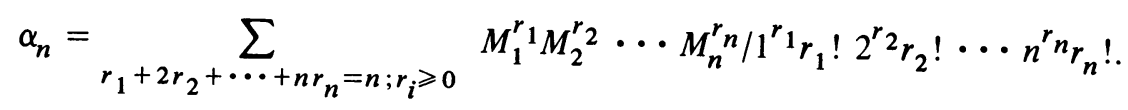

This discussion implies an interesting formal identity, which we mention in passing. If we consider (1) as a system of equations for $M_{1}, M_{2}, \ldots, M_{n}$, then Cramer's rule implies the following: If $A$ is the $n \times n$ matrix

$$
A=\left[\begin{array}{cccc}
1 & & & 1 \\
\alpha_{1} & 1 & & 2 \alpha_{2} \\
\alpha_{2} & \alpha_{1} & 1 & 3 \alpha_{3} \\
& \ldots & & \ldots \\
\alpha_{n-1} \alpha_{n-2} & \alpha_{n-3} & & n \alpha_{n}
\end{array}\right],
$$

then

$$
\operatorname{det}(A)=n \sum_{k=1}^{n} \frac{(-1)^{k-1}}{k} S_{k}
$$

where $S_{k}$ is given by (6).

We now assume certain properties of the sequences $\alpha_{0}, \alpha_{1}, \alpha_{2}, \ldots, M_{1}, M_{2}$, $M_{3}, \ldots$, and use them to derive the following lemma, which will form the basis of the discussion of asymptotic properties that follows.

LEMMA 1. Let $\alpha_{0}, \alpha_{1}, \alpha_{2}, \ldots$ be a sequence of positive numbers such that $\alpha_{0}=1$. Suppose that $M_{1}, M_{2}, M_{3}, \ldots$ is also a sequence of positive numbers, and that

$$
\sum_{k=1}^{n} \alpha_{n-k} M_{k}=n \alpha_{n}, \quad n \geqslant 1
$$

Put

$$
A_{n}=\sum_{k=1}^{n-1} \alpha_{k} \alpha_{n-k} / \alpha_{n}
$$

and assume that $A_{n} \rightarrow 0$ as $n \rightarrow \infty$. Then $M_{n} \sim n \alpha_{n}$. 
Proof. Because of the positivity, we have that

$$
M_{n} \leqslant \sum_{k=1}^{n} \alpha_{n-k} M_{k}=n \alpha_{n}, \quad n \geqslant 1
$$

Thus

$$
\begin{gathered}
\sum_{k=1}^{n-1} \alpha_{n-k} M_{k} \leqslant \sum_{k=1}^{n-1} \alpha_{n-k} \cdot k \alpha_{k}=\frac{n}{2} \sum_{k=1}^{n-1} \alpha_{n-k} \alpha_{k} \\
\sum_{k=1}^{n-1} \alpha_{n-k} M_{k} \leqslant \frac{n}{2} \alpha_{n} A_{n} .
\end{gathered}
$$

It follows that

$$
n \alpha_{n}=M_{n}+\sum_{k=1}^{n-1} \alpha_{n-k} M_{k} \leqslant M_{n}+\frac{n}{2} \alpha_{n} A_{n}, \quad M_{n} \geqslant n \alpha_{n}\left(1-\frac{1}{2} A_{n}\right) .
$$

Thus

$$
1-1 / 2 A_{n} \leqslant M_{n} / n \alpha_{n} \leqslant 1
$$

and so, $M_{n} / n \alpha_{n} \rightarrow 1$ as $n \rightarrow \infty$, since $A_{n} \rightarrow 0$ as $n \rightarrow \infty$. This completes the proof.

For the case of free groups of finite rank we also require the following lemma:

Lemma 2. Suppose that $s \geqslant 1$, and put

$$
A_{n}=\sum_{k=1}^{n-1}\left(\begin{array}{l}
n \\
k
\end{array}\right)^{-s}, \quad n \geqslant 1
$$

Then $A_{n} \rightarrow 0$ as $n \rightarrow \infty$.

Proof. We may assume that $n \geqslant 3$. Since $\left(\begin{array}{l}n \\ k\end{array}\right) \geqslant\left(\begin{array}{l}n \\ 2\end{array}\right)$ for $2 \leqslant k \leqslant n-2$, we have

$$
\begin{aligned}
A_{n} & =2 n^{-s}+\sum_{k=2}^{n-2}\left(\begin{array}{l}
n \\
k
\end{array}\right)^{-s} \leqslant 2 n^{-s}+(n-3)\left(\begin{array}{l}
n \\
2
\end{array}\right)^{-s} \\
& =2 n^{-s}+n^{-s}(n-3)\left(\frac{n-1}{2}\right)^{-s} .
\end{aligned}
$$

Now $(n-1) / 2 \geqslant 1$, since $n \geqslant 3$. It follows that

$$
A_{n} \leqslant 2 n^{-s}+n^{-s}(n-3)\left(\frac{n-1}{2}\right)^{-1} \leqslant 4 n^{-s} \text {. }
$$

Since $A_{n}$ is positive, the result follows.

We now use these lemmas to obtain our first asymptotic result:

THEOREM 2. Let $M_{r}(n)$ be the number of subgroups of index $n$ of the free group of rank $r$, where $r \geqslant 2$. Then

$$
M_{r}(n) \sim n \cdot n !^{r-1} .
$$

Proof. M. Hall's recurrence formula for $M_{r}(n)$ [2] states that

$$
\sum_{k=1}^{n}(n-k) !^{r-1} M_{r}(k)=n \cdot n !^{r-1}, \quad n \geqslant 1 .
$$


By Lemma 1 we need only show that if

$$
A_{n}=\sum_{k=1}^{n-1} k !^{r-1}(n-k) !^{r-1} / n !^{r-1}=\sum_{k=1}^{n-1}\left(\begin{array}{l}
n \\
k
\end{array}\right)^{1-r}
$$

then $A_{n} \rightarrow 0$ as $n \rightarrow \infty$. But this is the content of Lemma 2, since $r-1 \geqslant 1$. In fact, inequality (8) implies that

$$
1-\frac{2}{n^{r-1}} \leqslant \frac{M_{r}(n)}{n \cdot n !^{r-1}} \leqslant 1, \quad n \geqslant 3 .
$$

This completes the proof.

The formal power series

$$
y=\sum_{n=0}^{\infty} n ! x^{n}=1+x+\cdots
$$

is worth special consideration. Put

$$
1 / y=\sum_{n=0}^{\infty} c_{n} x^{n}=1-x+\cdots
$$

We have

$$
\begin{gathered}
x y=\sum_{n=0}^{\infty} n ! x^{n+1}, \quad x y^{\prime}+y=\sum_{n=0}^{\infty}(n+1) ! x^{n}=(y-1) / x, \\
x y^{\prime} / y+1=1-1 / y=x-c_{2} x^{2}-\cdots
\end{gathered}
$$

Hence

$$
x y^{\prime} / y=-c_{2} x-c_{3} x^{2}-\cdots .
$$

Coupled with the previous discussion, we get

THEOREM 3. Let $c_{n}$ be the coefficient of $x^{n}$ in the reciprocal of the formal power series $\Sigma_{n=0}^{\infty} n ! x^{n}$. Then $-c_{n+1}$ is the number of subgroups of index $n$ of the free group of rank 2 .

We go on to other groups. Let $\tau_{p}(n)$ denote the number of homomorphisms of the cyclic group $C_{p}$ of order $p$ into the symmetric group $S_{n}$. Thus $\tau_{p}(n)$ is the number of elements of $S_{n}$ whose order divides $p$. The generating function for $\tau_{p}(n) / n$ ! is given by

$$
\sum_{n=0}^{\infty} \frac{\tau_{p}(n)}{n !} x^{n}=\exp \left(\sum_{d \mid p} \frac{x^{d}}{d}\right) .
$$

If $p$ is a prime, $\tau_{p}(n)$ is given by

$$
\tau_{p}(n)=\sum_{0 \leqslant r \leqslant n / p} n ! / r !(n-r p) ! p^{r}
$$

and

$$
\sum_{n=0}^{\infty} \frac{\tau_{p}(n)}{n !} x^{n}=\exp \left(x+\frac{x^{p}}{p}\right)
$$


The importance of these numbers stems from the work of I. M. S. Dey [1] who showed that if $M_{n}$ is the number of subgroups of index $n$ of the free product $C_{p_{1}} * C_{p_{2}} * \cdots$ * $C_{p_{k}}$, where $p_{i}$ is either $\infty$ or an integer $\geqslant 2$, then $M_{n}$ satisfies the recurrence (1), with

$$
\alpha_{n}=\tau_{p_{1}}(n) \tau_{p_{2}}(n) \cdots \tau_{p_{k}}(n) / n !
$$

Of course, $\tau_{\infty}(n)=n !$.

When $p$ is prime, $\tau_{p}(n)$ is most easily calculated by the recurrence formula

$$
\tau_{p}(n+1)=\tau_{p}(n)+(p-1) !\left(\begin{array}{c}
n \\
p-1
\end{array}\right) \tau_{p}(n-p+1), \quad n \geqslant p-1
$$

with the initial conditions

$$
\tau_{p}(0)=\tau_{p}(1)=\cdots=\tau_{p}(p-1)=1 .
$$

The asymptotic behavior of $\tau_{p}(n)$ for $p$ prime was determined by L. Moser and $M$. Wyman in [3], by means of the generating function (11). They showed that

$$
\tau_{p}(n) \sim K_{p} \exp \left(\frac{p-1}{p} n \log n-\frac{p-1}{p} n+n^{1 / p}\right)
$$

where

$$
K_{2}=2^{-1 / 2} e^{-1 / 4}, \quad K_{p}=p^{-1 / 2}, \quad p>2 .
$$

It follows from (13), (14), and Stirling's formula that

$$
\tau_{2}(n) \tau_{3}(n) / n ! \sim K \exp \left(\frac{n}{6} \log n-\frac{n}{6}+n^{1 / 2}+n^{1 / 3}-\frac{1}{2} \log n\right),
$$

where $K=\left(12 \pi e^{1 / 2}\right)^{-1 / 2}$.

Of particular interest is the case $\Gamma=C_{2} * C_{3}$, the classical modular group. We wish to show that in this case

$$
M_{n} \sim \tau_{2}(n) \tau_{3}(n) /(n-1) !
$$

This is rather more difficult than the problem for free groups of finite rank, and the asymptotic properties of the coefficients $\tau_{2}(n), \tau_{3}(n)$ come into play. In a welldefined sense, this is the most difficult case. The coefficients in the recurrence formula grow least rapidly, corresponding to the fact that $\Gamma$ has the smallest hyperbolic area of all noncompact Fuchsian groups. Further comments on this point will be made later on.

The basic problem will be to show that if

$$
\alpha_{n}=\tau_{2}(n) \tau_{3}(n) / n !
$$

then

$$
A_{n}=\sum_{k=1}^{n-1} \alpha_{k} \alpha_{n-k} / \alpha_{n} \rightarrow 0 \quad \text { as } n \rightarrow \infty
$$


The discussion that follows is devoted to this end.

The asymptotic formula (15) implies that

$$
A_{n}=O\left(\sum_{1 \leqslant k \leqslant n / 2} \exp (\theta(k)+\theta(n-k)-\theta(n))\right),
$$

where

$$
\theta(n)=\frac{n}{6} \log n+n^{1 / 2}+n^{1 / 3} .
$$

(Here we have used the symmetry of the sum, and the facts that the terms in the exponent corresponding to $-n / 6$ disappear, and that $n / k(n-k)$ is bounded by an absolute constant for $1 \leqslant k \leqslant n-1$.)

Consider

$$
\theta(x)=\frac{x}{6} \log x+x^{1 / 2}+x^{1 / 3}
$$

Then a brief calculation shows that

$$
36 x^{5 / 3} \theta^{\prime \prime}(x)=6 x^{2 / 3}-9 x^{1 / 6}-8,
$$

and that $\theta^{\prime \prime}(x) \geqslant 0$ for $x \geqslant 6.17250 \ldots$. Hence $\theta^{\prime \prime}(x) \geqslant 0$ for $x \geqslant 7$, and it follows that $\theta^{\prime}(x)$ is monotone increasing for $x \geqslant 7$.

Now consider the inequality

$$
\theta(k+1)+\theta(n-k-1) \leqslant \theta(k)+\theta(n-k) .
$$

This will hold if and only if

$$
\theta(k+1)-\theta(k) \leqslant \theta(n-k)-\theta(n-k-1) .
$$

We have

$$
\begin{array}{cl}
\theta(k+1)-\theta(k)=\theta^{\prime}\left(k+\sigma_{1}\right), & 0 \leqslant \sigma_{1} \leqslant 1, \\
\theta(n-k)-\theta(n-k-1)=\theta^{\prime}\left(n-k-1+\sigma_{2}\right), & 0 \leqslant \sigma_{2} \leqslant 1 .
\end{array}
$$

Assume that

$$
7 \leqslant k \leqslant 1 / 2 n-1 \text {. }
$$

Then $k+1 \leqslant n-k-1$, and using the fact that $\theta^{\prime}(x)$ is monotone increasing for $x \geqslant$ 7 , we get

$$
\begin{aligned}
\theta(k+1)-\theta(k) & =\theta^{\prime}\left(k+\sigma_{1}\right) \leqslant \theta^{\prime}(k+1) \leqslant \theta^{\prime}(n-k-1) \\
& \leqslant \theta^{\prime}\left(n-k-1+\sigma_{2}\right)=\theta(n-k)-\theta(n-k-1) .
\end{aligned}
$$

It follows that (17) holds, provided that $k$ satisfies (18). We state the consequence of this result as a lemma.

Lemma 3. The function $\theta(k)+\theta(n-k)$ satisfies

$$
\theta(k)+\theta(n-k) \leqslant \theta(7)+\theta(n-7), \quad 7 \leqslant k \leqslant n / 2 .
$$


We also remark that if $k$ remains bounded, then

$$
\theta(k)+\theta(n-k)-\theta(n)=-\frac{k}{6} \log n+O(1) .
$$

We can now prove

Lemma 4. Let $A_{n}=\sum_{k=1}^{n-1} \alpha_{k} \alpha_{n-k} / \alpha_{n}$, where $\alpha_{n}=\tau_{2}(n) \tau_{3}(n) / n !$. Then $A_{n}=$ $O\left(n^{-1 / 6}\right)$, so that $A_{n} \rightarrow 0$ as $n \rightarrow \infty$.

Proof. By Lemma 3,

$$
\begin{aligned}
A_{n}= & O\left(\sum_{1 \leqslant k \leqslant 6} \exp (\theta(k)+\theta(n-k)-\theta(n))\right) \\
& +O\left(\sum_{7 \leqslant k \leqslant n / 2} \exp (\theta(7)+\theta(n-7)-\theta(n))\right) .
\end{aligned}
$$

We have

$$
\sum_{1 \leqslant k \leqslant 6} \exp (\theta(k)+\theta(n-k)-\theta(n))=\sum_{1 \leqslant k \leqslant 6} \exp \left(-\frac{k}{6} \log n+O(1)\right)=O\left(n^{-1 / 6}\right)
$$

and

$$
\sum_{7 \leqslant k \leqslant n / 2} \exp (\theta(7)+\theta(n-7)-\theta(n))=O\left(n \exp \left(-\frac{7}{6} \log n+O(1)\right)\right)=O\left(n^{-1 / 6}\right)
$$

The result now follows.

Lemmas 1 and 4 now imply our desired result:

THEOREM 4. Let $M_{n}$ denote the number of subgroups of index $n$ of the classical modular group $\Gamma$. Then

$$
M_{n} \sim \tau_{2}(n) \tau_{3}(n) /(n-1) ! \sim K \exp \left(\frac{n}{6} \log n-\frac{n}{6}+n^{1 / 2}+n^{1 / 3}+\frac{1}{2} \log n\right),
$$

where $K=\left(12 \pi e^{1 / 2}\right)^{-1 / 2}$.

Precisely the same discussion applies to the more general case when $M_{n}$ is the number of subgroups of index $n$ of the free product $C_{p_{1}} * C_{p_{2}} * \cdots * C_{p_{k}}$, with one exception. The corresponding result is that

$$
M_{n} \sim \tau_{p_{1}}(n) \tau_{p_{2}}(n) \cdots \tau_{p_{k}}(n) /(n-1) !,
$$

where $\tau_{p}(n)$ is the number of homomorphisms of $C_{p}$ into $S_{n}$. The exception occurs for $C_{2} * C_{2}$. The difficulty here is that $\tau_{2}(n)^{2} / n$ ! does not grow fast enough; in fact,

$$
\tau_{2}(n)^{2} / n ! \sim K \exp \left(2 n^{1 / 2}-\frac{1}{2} \log n\right)
$$

where $K=(8 \pi e)^{-1 / 2}$. The exception is quite natural in view of the fact that this is the only group of the form $C_{p_{1}} * C_{p_{2}} * \cdots * C_{p_{k}}$ which does not have a representation as a Fuchsian group, since it would correspond to one of genus 0 , with a single parabolic generator and 2 elliptic generators of order 2; and so would have zero hyperbolic area, which is not possible. 


\section{$M(N)$ is the number of subgroups of the classical modular group of index $N$}

$\mathbf{N}$

M( N )

1

1

4

8

5

22

42

40

120

$2 \in 5$

286

$7 \in 4$

1725

2198

5168

$1 \approx 144$

17034

37702

$88 \subseteq 58$

136584

$28827 \mathrm{C}$

6E2572

1118996

$2 \geq 064 € 4$

5428800

9 4 C 517

19103988

44701696

80904113

163344502

$37924 \subseteq 288$

711598944

1434840718

$3305 \subseteq 97062$

6391673638

1 2921 383032

29611074174

58602591708

119001063028

$271 \geq 31133136$

547872065136

1119204224 EE 6

2541384297716

5219606253184

10733985041978

24300914061436

50635071045768

104875736986272

$23 \in \subseteq 34212877684$

$4 \subseteq \$ 877 \subseteq 70 \subseteq \& 566 \mathrm{C}$
N $\quad M(N)$

511042904230435308

52 23532ฐ8168183056

$53 \quad 5018827370579404$

$54 \quad 10547597621517112$

$55 \quad 23788180556758856$

5651219574162595680

57 $108420140150 \$ 58464$

58 244556725280402557

$59 \quad 531051678812968744$

$60 \quad 1132058252012247536$

612555221387154759289

62 559115460SOE7446054

$63 \quad 12000605074451550160$

$64 \quad 27117552811153855680$

C5 59749471015816115222

EE 129096123267775868166

$67 \quad 292152183906195140230$

$68 \quad 647820636135303527128$

$69140868030 \leqslant 233863887966$

70 3ISJES13654SE30S148284

$71 \quad 7123474185205298268592$

$7215585 \varepsilon 66806530333864208$

$7=\quad 3540839 \subseteq 627074036560816$

$74 \quad 7 \subseteq 4117969265 \varepsilon 985 \subseteq 301294$

$75 \quad 174786866873511715532628$

$76 \quad 3 \subseteq 7988303771373972895968$

$77 \quad 897183482867489002743454$

$78 \quad 1586021323730942427050260$

$79453333701587714366 \in 107784$

Q0 $102691900242876408 \varepsilon 3655792$

$8122858848125 \subseteq 75541396523980$

e2 52311:44432011656725753204

83 119045150159430509536306820

\&4 2E640803840S645S23245611632

85611297865534522328810726884

\&€ $\quad 1 \geq \varsigma 7257675120158769 C 8 \subseteq 798632$

$87 \quad 3143030617470775207826061556$

$8872319>0266681482552134898592$

$89 \quad 16599371653596557362869244032$

@0 $\quad 375263 C 7>C>214870497477862098$

918 $8650_{1501222729298577623800376}$

S2 1 CS5611848454364S\$4086184\$1008 453310522764485571147172334106 1049023788372134074385428390460 2427029113004378647451505343600 $5538>779,065385705215 \varsigma 73 \Xi 3058368$ 12854913784226053720296237333900 2S?531810615148067506978864-7212 68435931318931500012779730780508 15925 S5 2010504751878902805384624

To illustrate how the problem depends on the hyperbolic area, assume that each $p_{i}$ is $\infty$ or a prime. Let the multiplicity of $\infty$ be $t-1(t \geqslant 1)$, and let the remaining $p_{i}$ be denoted by $e_{i}(1 \leqslant i \leqslant s)$, so that $t+s-1=k$. Then if

$$
\alpha_{n}=\tau_{p_{1}}(n) \tau_{p_{2}}(n) \cdots \tau_{p_{k}}(n) / n !,
$$

formula (13) implies that

$$
\alpha_{n} \sim K \exp \left(H n \log n-H n+\sum_{i=1}^{k} n^{1 / e_{i}}+\left(\frac{1}{2} t-1\right) \log n\right),
$$


where $K$ is a constant which need not be specified, and

$$
H=t-2+\sum_{i=1}^{s}\left(1-\frac{1}{e_{i}}\right) .
$$

Thus the growth of $\alpha_{n}$ depends upon $H$, and apart from a constant factor, $H$ is just the hyperbolic area of $C_{p_{1}} * C_{p_{2}} * \cdots * C_{p_{k}}$.

In conclusion we append a table of $M_{n}$ for $1 \leqslant n \leqslant 100$, where $M_{n}$ is the number of subgroups of index $n$ of $\Gamma=C_{2} * C_{3}$. The table was computed in a negligible amount of time using residue arithmetic by means of the recurrence formula (1), with $\alpha_{n}=\tau_{2}(n) \tau_{3}(n) / n$ !. The approach to 1 of the ratio $M_{n} / n \alpha_{n}$ is quite slow, and agrees well with the estimate

$$
\left|1-M_{n} / n \alpha_{n}\right|=O\left(n^{-1 / 6}\right)
$$

derived before.

A useful check on the computation is that if $n=p^{e}$, where $p$ is a prime and $n>3$, then $M_{n} \equiv 0 \bmod p$. This is so because $\Gamma$ contains no normal subgroups of index $n$.

National Bureau of Standards

Washington, D. C. 20234

1. I. M. S. DEY, "Schreier systems in free products," Proc. Glasgow Math. Assoc., v. 7, 1965, pp. 61-79. MR 32 \#5718.

2. M. HALL, JR., "Subgroups of finite index in free groups," Canad. J. Math., v. 1, 1949, pp. 187-190. MR 10, 506.

3. L. MOSER \& M. WYMAN, “On solutions of $x^{d}=1$ in symmetric groups," Canad. J. Math., v. 7, 1955, pp. 159-168. MR 16, 904. 